\title{
RNA-seq analysis of prostate cancer in the Chinese popu- lation identifies recurrent gene fusions, cancer-associated long noncoding RNAs and aberrant alternative splicings
}

Shancheng Ren, Zhiyu Peng, Jian-Hua Mao, Yongwei Yu, Changjun Yin, Xin Gao, Zilian Cui, Jibin Zhang, Kang Yi, Weidong Xu, Chao Chen, Fubo Wang, Xinwu Guo, Ji Lu, Jun Yang, Min Wei, Zhijian Tian, Yinghui Guan, Liang Tang, Chuanliang Xu, Linhui Wang, Xu Gao, Wei Tian, Jian Wang, Huanming Yang, Jun Wang, Yinghao Sun

Cell Research (2013) 23:732. doi:10.1038/cr.2013.61; published online 2 May 2013

Addendum to: Cell Research (2012) 22:806-821. doi:10.1038/cr.2012.30; published online 21 February 2012

The RNA-seq data of 14 pairs of prostate cancer in this paper can be download via the following link.

http://www.ebi.ac.uk/arrayexpress/experiments/E-MTAB-567/samples/ 